\title{
Linguistes, activistes et locuteurs : trois terrains croisés (vepse, tabarquin, croate molisain)
}

Linguists, Activists and Language Speakers: Three Crossed Fields (Vepsians, Tabarquins and Molise Croatians)

Keeleteadlased, aktivistid ja keekekandjad: kolm ristitud välitööd (vepslased, tabarkiinid ja Molise horvaadid)

\section{Ksenija Djordjević-Léonard}

\section{(2) OpenEdition}

\section{Journals}

Édition électronique

URL : https://journals.openedition.org/efo/9951

DOI : $10.4000 /$ efo.9951

ISSN : 2275-1947

\section{Éditeur}

INALCO

\section{Référence électronique}

Ksenija Djordjević-Léonard, «Linguistes, activistes et locuteurs : trois terrains croisés (vepse,

tabarquin, croate molisain) », Études finno-ougriennes [En ligne], 49-50 | 2018, mis en ligne le 19 février 2019, consulté le 06 octobre 2022. URL : http://journals.openedition.org/efo/9951 ; DOI : https:// doi.org/10.4000/efo.9951

Ce document a été généré automatiquement le 6 octobre 2022.

\section{(c) (†) (8)}

Creative Commons - Attribution - Pas d'Utilisation Commerciale 4.0 International - CC BY-NC 4.0 https://creativecommons.org/licenses/by-nc/4.0/ 


\section{Linguistes, activistes et locuteurs : trois terrains croisés (vepse, tabarquin, croate molisain)}

Linguists, Activists and Language Speakers: Three Crossed Fields (Vepsians, Tabarquins and Molise Croatians)

Keeleteadlased, aktivistid ja keekekandjad: kolm ristitud välitööd (vepslased, tabarkiinid ja Molise horvaadid)

Ksenija Djordjević-Léonard

\section{Introduction}

1 Nous proposons, dans cette contribution, de croiser trois expériences récentes de terrain que nous avons réalisées dans le contexte minoritaire européen: les terrains vepse, tabarquin et croate molisain. Le premier de ces terrains a pris la forme d'un séjour au sein de la communauté vepse de la république russe de Carélie, en avril-mai 2013. Le second a été réalisé en mai 2014, sur l'île sarde de San Pietro, peuplée majoritairement par les Tabarquins. Le troisième terrain s'est déroulé également en Italie, dans le Molise, dans des villages habités par la minorité croate, en avril $2016^{1}$. Si ces trois exemples relèvent de situations différentes, sur le plan historique, politique et démographique, il nous semble qu'ils sont susceptibles d'illustrer de manière heuristique les différentes facettes du micro-aménagement linguistique, pensé et conduit essentiellement par la société civile. L'approche réflexive du/des terrain(s) ${ }^{2}$ que nous proposons ici sera donc alimentée par l'observation et l'écoute des locuteurs, dont certains sont devenus des activistes, in situ, au sein des trois communautés mentionnées.

Dans ce qui suit, nous définirons, dans un premier temps, la (socio)linguistique de terrain: sa raison d'être, ses caractéristiques, ses méthodes. L'aménagement linguistique "de par en bas", ou encore "à mi-palier», seront définis dans la 
deuxième partie, à travers notamment une réflexion sur les locuteurs et leur implication dans le travail de documentation, de sauvegarde, de re-fonctionnalisation ou de revitalisation de leurs langues. Enfin, nous relaterons notre expérience de terrain chez les Vepses, les Tabarquins et les Croates du Molise, afin d'illustrer la synergie possible entre les linguistes, les activistes et les locuteurs, qui est, selon nous, l'une des clés de réussite d'un terrain sociolinguistique, et en particulier d'un terrain orienté, comme dans notre cas, vers les langues vulnérables ou menacées de disparition.

\section{La (socio)linguistique de terrain}

3 La sociolinguistique est une discipline de terrain, une discipline empirique qui fonde ses théories sur l'observation des réalités langagières. Comme le soulignent les sociologues S. Beaud et F. Weber, cette notion «à la mode » est de plus en plus utilisée dans les sciences sociales comme "une manière commode et creuse d'afficher une proximité avec ses concitoyens et de combler magiquement le fossé entre les “élites" et le "peuple" " (Beaud et Weber, 2003, p. 7), comme si le fait d'aller sur le terrain apportait immanquablement la dose de crédibilité nécessaire à des propos émanant d'observateurs externes "concernés » à divers titres (décideurs politiques, entrepreneurs, chercheurs, etc.). Pour certains sociolinguistes, le terrain est le point de départ de tout travail sur les langues, et aussi la consécration de celui-ci. Il est souvent considéré comme une étape incontournable dans le parcours d'un chercheur, et même d'un étudiant en sociolinguistique : l'enquête de terrain doit compléter et étayer la réflexion scientifique - une réflexion qui, à son tour, se nourrit et s'inspire de ce même terrain.

4 Si les chercheurs anglo-saxons insistent sur l'idée du travail sur le terrain ( fieldwork »), et les chercheurs russes parlent d'expédition (« экспЕдиция »), le terme le plus souvent utilisé par nos administrations universitaires en France est celui de mission. Cette floraison des termes, plus ou moins impropres, nous semble mal désigner la réalité complexe du métier de chercheur en sciences humaines, « en dehors du bureau». Les notions de mission et d'expédition, en particulier, comportent des connotations négatives, comme si le chercheur quittait le confort de sa position institutionnelle pour se rendre sur un lieu exotique et lointain, poussé par un objectif bien précis, comme investi d'un «travail civilisateur" à accomplir, grâce à la « science » dont il serait le détenteur, aux yeux du grand public. Or, les terrains ne sont pas nécessairement lointains ni forcément toujours très exotiques. Ils sont là autour de nous, nous les choisissons, nous les créons, nous les faisons exister, par le regard que nous y portons, et l'analyse que nous en donnons. Le point de vue crée l'objet, disait encore Saussure, et la sociolinguistique qui s'est positionnée en partie en opposition par rapport à la linguistique structurale, n'a pas démenti cette affirmation à ce jour bien au contraire.

De la même manière, les termes d'enquête et d'informateur semblent, si ce n'est impropres, du moins exagérés, avec leurs connotations administratives ou même policières. Comme si chaque situation cachait volontairement son vrai visage, que seul le chercheur, grâce à ses investigations - voilà encore un terme, du domaine du journalisme, qui n'est pas absent de nos travaux -, peut réussir à démasquer et à percer à jour. Or, le terrain n'est autre que la réalité d'un espace, d'un lieu, qu'il soit devant nos yeux ou à l'autre bout du monde. C'est la méthodologie que le chercheur applique 
qui donne du sens aux observations. Cette méthodologie, le chercheur la construit longuement avant de partir, et l'adapte au quotidien en contact avec les gens, les sujets, les interlocuteurs.

6 Dans notre discipline - la sociolinguistique - la méthodologie prend forme le plus souvent de questionnaires, d'entretiens de préférence semi-directifs, d'observation, directe ou participante, ou d'un mélange de tout cela, en fonction des objectifs fixés au départ, et des aléas de la vie de chercheur. On a beau s'appliquer à soi-même des conseils que l'on donne à nos étudiants (choisir un thème qui nous passionne, un domaine dans lequel nous sommes compétents, un objectif réalisable, une méthode adaptée, etc.), on n'est jamais à l'abri d'un imprévu. Les mauvais choix faits au départ, les mauvaises périodes retenues, la non disponibilité des informateurs, etc. font que le travail envisagé prend parfois une forme tout à fait inattendue. En réalité, cela peut être très déconcertant : préparer les questions et les questionnaires, les traduire s'il le faut, se déplacer... et ne pas toujours pouvoir faire l'enquête, car les informateurs ont autre chose à faire le jour du rendez-vous, ou c'est l'administration locale qui décide qu'un spectacle folklorique est susceptible de nous intéresser davantage qu'une discussion avec les locuteurs de telle ou telle langue que nous sommes venus observer dans ses pratiques langagières quotidiennes, ou par élicitation. Ces chants et ces danses folkloriques sont généralement très beaux, et les costumes forcent l'admiration, sans nul doute... mais cela ne correspond pas forcément à nos objectifs de recherche, et parfois on peut même avoir l'impression que ceux-ci sont de toute façon tout à fait secondaires pour nombre de nos partenaires locaux, y compris universitaires. Là aussi, le conseil donné aux étudiants - «ne vous découragez surtout pas » - reste finalement le meilleur vade-mecum. On n'est pas toujours prêt à accepter l'idée (en tous cas, sur le moment) que derrière le désespoir de ne pas pouvoir réaliser l'enquête qui était initialement prévue se cache parfois un terrain encore plus passionnant, encore plus riche, encore plus inattendu, que le chercheur invétéré transformera par son regard et ses interprétations, son propre bagage intellectuel et théorique, en une réalité captivante.

7 Le terrain, on l'aura compris, pour les sociolinguistes, est plus qu'une enquête, c'est un lieu d'échange et de rencontres, un lieu où se croisent le linguiste et les locuteurs, un lieu qui se déroule dans l'interaction. Le chercheur questionne, interroge, recoupe les informations, fait parler les gens, les écoute surtout, même lorsqu'ils ne répondent pas à ses questions précises, minutieusement préparées avant de partir. Il les écoute même quand ils décident de renverser les rôles et de lui imposer leurs propres règles du jeu, sans jamais s'arrêter de filmer, photographier et enregistrer les bribes de cette réalité qui lui a été donnée à voir, et sans jamais oublier ce qu'il est venu chercher : " une expérience que les livres et que les supports d'information-les médias du savoir-ne peuvent aucunement nous donner", autrement dit "une expérience sensible, dans l'acception philosophique du terme (l'empirisme), une sensitivité et du sens » (Léonard, 2014, p. 64). Ce même chercheur sait qu'en rentrant chez lui, dans son bureau, quand il aura pris ses distances avec le terrain, ces matériaux vont commencer une nouvelle vie, et même si une partie du corpus recueilli ne lui servira jamais, le peu dont il arrivera à retirer la substantifique moelle, sera déjà précieux.

8 Avant de relater nos propres expériences de terrain, nous présenterons, dans la section suivante, le prisme à travers lequel nous proposons de les observer: la place, le rôle 
et l'action des locuteurs, quelles que soient leurs compétences, dans l'aménagement linguistique local.

\section{Aménagement linguistique « de par en bas " ou « à mi-palier » : lorsque les locuteurs deviennent activistes}

Dans la littérature sociolinguistique consacrée à la gestion des langues, on fait de plus en plus la distinction entre un aménagement linguistique « de par en haut " (démarche descendante), «de par le bas» (démarche ascendante) et à «mi-palier». En ce qui concerne ce dernier, il est principalement mené par les intellectuels - enseignants, chercheurs ou travailleurs culturels - qui forment ce que l'on peut appeler l'élite locale. Dans le cas de micro-communautés linguistiques, comme celles qui nous intéressent ici, si l'État assure une protection, parfois pour des raisons historiques, à la minorité et sa langue - protection d'ailleurs souvent symbolique -, l'essentiel du travail est réalisé par la société civile, autrement dit par les activistes, souvent locuteurs plus ou moins actifs de la langue. Ils peuvent donc jouer un rôle analogue à celui des informateurs, pour le linguiste sur le terrain, et l'aider à collecter les données, qu'elles soient linguistiques ou sociolinguistiques.

On attend généralement d'un bon informateur qu'il soit également un bon locuteur. Cette condition est évidement encore plus nécessaire dans le cas d'un terrain à proprement parler linguistique (travail sur la langue elle-même). Cependant, même dans le cas d'un terrain sociolinguistique, un locuteur actif est souvent plus proche de la langue, plus conscient des conditions psycho-sociales de son usage. Mais encore faut-il qu'il ait véritablement envie de travailler avec le linguiste : les deux ne vont pas forcément ensemble, la compétence linguistique ne va pas de pair avec une sensibilité et une volonté de travailler avec les chercheurs.

Un chercheur-linguiste sur le terrain rencontre tout un éventail de locuteurs différents. Dans leur article publié dans la revue Faits de langues, à travers une réflexion sur les langues en danger, M. Bert et C. Grinevald proposent sept profils typiques de locuteurs que nous allons résumer de manière succincte :

1. Locuteurs traditionnels (locuteurs qui utilisent encore la langue de façon régulière, ou qui viennent à peine de cesser de la parler) ;

2. Semi-locuteurs (ces locuteurs se caractérisent généralement par une bonne compétence de compréhension, une compétence de production variable, et une pratique irrégulière) ;

3. Sous-locuteurs (les compétences passives de ces locuteurs sont importantes, leurs compétences actives limitées) ;

4. Anciens locuteurs (il s'agit de personnes qui ont « oublié » ou cessé de parler la langue, mais une réactivation des compétences reste possible sous certaines conditions);

5. Locuteurs fantômes (personnes qui refusent d'admettre qu'elles ont des compétences dans une langue menacée, qui ont développé une attitude négative vis-à-vis de celle-ci, dans un contexte d'aliénation culturelle souvent avancée) ;

6. Néo-locuteurs (personnes qui ont appris la langue, qui ont une compétence linguistique assez variable, mais leur attitude vis-à-vis de la langue reste positive) ;

7. Derniers locuteurs (comme le nom l'indique, il s'agit de derniers représentants de la communauté linguistique) (Bert et Grinevald, 2010, p. 125-129). 
Il va de soi que cette typologie dépend grandement de la configuration sociolinguistique de chaque territoire observé, du parcours de vie de chaque locuteur, et en tant que telle, elle est variable : « ces évolutions, qui influent sur la composition de la population des locuteurs et sur leurs attitudes envers la langue et les projets en cours, peuvent également entraîner des changements dans les relations entre locuteurs et linguistes » (Bert et Grinevald, 2010, p. 131).

En effet, au sein d'une même communauté linguistique, des locuteurs réagissent différemment: certains peuvent exprimer clairement l'envie de recevoir une aide venue de l'extérieur (de linguistes par exemple) pour s'impliquer dans un travail de valorisation du patrimoine linguistique et culturel, tandis que pour d'autres, il s'agira d'une motivation spontanée, ou même pour certains, d'un travail inutile auquel ils ne vont pas souhaiter participer. Certains rejetteront toute aide extérieure, mais accepteront des initiatives locales, ou bien l'inverse. Puis, sur le terrain, on peut rencontrer tout simplement des locuteurs, tous âges confondus, qui parlent la langue naturellement, sans jamais se poser la question de l'importance de cette pratique pour nous, les linguistes, et pour la science... Tout cela fait partie des découvertes du linguiste sur le terrain; il s'agit de ces facettes de la réalité que l'on peut difficilement aborder et imaginer à l'avance.

14 Nous observerons, dans la section suivante, le rôle des locuteurs-activistes dans l'aménagement linguistique sur les trois terrains retenus dans le cadre de cette contribution, en portant notre regard surtout sur les activités de revitalisation, qui consistent à accompagner les communautés sur le chemin de la sauvegarde de leurs pratiques langagières. La condition sine qua non de ce travail est que les locuteurs soient demandeurs d'une telle opération de "sauvetage " ou d'une réhabilitation du statut et des fonctions de la langue, et des formes que celle-ci prendra dans le cadre de la société contemporaine. Dans un numéro de la revue Langage \& Société, publié en 2013, dirigé par James Costa, et consacré à la revitalisation, ce sociolinguiste se demande «pourquoi certaines personnes s'emparent-elles d'une langue à un moment donné pour la transformer en un objet de mobilisation?» (Costa, 2013, p. 9). Si l'on observe les repositionnements entre les majorités et les minorités suite aux grands bouleversements de la fin du xxe siècle, on se rend compte que les communautés réagissent différemment: soit elles évitent de monter sur le devant de la scène avec leurs revendications, soit elles se mobilisent, et cette mobilisation peut prendre les formes de l'activisme. La revitalisation est l'une des formes de cet activisme. Elle implique une collaboration étroite entre différents acteurs, et, a priori, ne semble pas relever des compétences de simples citoyens. Cependant, notre expérience sur des terrains divers nous a permis de voir qu'en l'absence d'autres moyens, ou face à l'indifférence des structures "de par en haut", les locuteurs déploient souvent des efforts remarquables pour mettre leurs talents au service de la survie et de la protection de leur langue. Voyons à présent comment, à partir de trois études de cas.

\section{Trois terrains croisés}

\section{Terrain vepse}

15 En 2013, grâce à un projet de recherche franco-russe conçu par l'université de Bordeaux-Montaigne sur les marges linguistiques, Jean Léo Léonard et nous-même 
avons souhaité travailler sur le vepse, une langue finno-ougrienne en situation de forte minoration, parlée en République de Carélie et dans deux oblasts du nord-ouest de la Fédération de Russie. Le projet interuniversitaire n'a pas abouti - comme cela arrive parfois pour des raisons administratives, comptables et budgétaires qui dépassent de loin le chercheur en tant qu'individu - mais les préparatifs ayant bien avancé, nous avons décidé de partir sur le terrain, sans attendre une éventuelle nouvelle étape de soumission qui, en cas de réponse positive, nous aurait certainement un peu facilité les choses sur le plan financier. Mais conformément au dicton qui dit que quand on aime, on ne compte pas, pour nous, la découverte du vepse était le début d'une véritable aventure sociolinguistique, et nous avons donc réalisé ce terrain en chercheurs indépendants.

16 La langue vepse ne compte aujourd'hui qu'un peu plus de 3000 locuteurs en Russie, ce qui la place parmi les langues déjà sérieusement en danger, selon la terminologie de l'Unesco. Située à l'extrémité d'un continuum dialectal qui va du finnois oriental au vepse, en passant par d'autres variétés linguistiques, plus ou moins individuées, comme le carélien, le lude et le livvi, cette langue se trouve aujourd'hui prise dans ce que nous avons appelé une dynamique sérielle d'altérités vis-à-vis du russe, du finnois et de ses langues collatérales (Eloy, 2004). Le vepse, le carélien et le finnois bénéficient d'une certaine reconnaissance patrimoniale en Carélie - ce qui n'est pas le cas des variétés livvi et lude-mais, en réalité, cette reconnaissance symbolique n'a pas empêché l'assimilation des Vepses par le groupe majoritaire. L'assimilation a commencé bien avant l'éclatement de l'URSS, et se poursuit inexorablement depuis, en entrainant non seulement les Vepses, mais tous les peuples minoritaires, dans une chute vertigineuse, du point de vue de l'attrition sociolinguistique. Or, de nos jours, même si la langue est peu présente dans la sphère publique, l'administration, l'enseignement, le domaine informatique et culturel, elle est tout de même visible dans la société. On pourrait presque dire qu'elle est davantage visible que par le passé, et notamment l'époque où les locuteurs étaient plus nombreux. Les facteurs qui, à notre sens, ont contribué à rendre le vepse davantage visible à l'époque moderne, en dépit de sa forte attrition sociolinguistique, sont le réveil de la conscience nationale, qui a marqué le monde finno-ougrien à la chute du communisme, le soutien des instances officielles, et bien sûr, le travail des aménageurs sur la revitalisation de la langue, qui œuvrent à sa valorisation en tant que vecteur de création culturelle.

17 C'est précisément avec les aménageurs que nous avons voulu travailler lors de notre séjour de terrain en avril-mai 2013, et c'est auprès d'eux ou avec eux que nous avons réalisé toute une série d'entretiens, tout en collectant d'autres corpus sur leur action travail qui a abouti à une riche documentation portant sur ce que les acteurs sur place appellent la "mobilisation ethnique ». Ce séjour nous a permis de voir comment se réalise in vivo l'aménagement linguistique "de par en bas » ou "à mi-palier ». Nous sommes restés deux semaines en Carélie, mais de cette mission relativement courte, nous sommes revenus avec une douzaine d'heures d'enregistrements et de films (entretiens sociolinguistiques, conversations spontanées dans des clubs de discussion, lectures de textes et de poèmes, récits de vie, morceaux de chants traditionnels, et, comme nous l'avons mentionné plus haut, une documentation importante). Nous avons beaucoup côtoyé les enseignants, les chercheurs ou les travailleurs culturels, donc une certaine élite vepse. Ils sont eux-mêmes locuteurs plus ou moins actifs de la langue, écrivent et lisent en vepse, et mènent un véritable combat pour sa sauvegarde. 
Ils « couvrent » vraiment tous les milieux dans lesquels se joue la survie de la langue : recherche (chercheurs qui travaillent sur les peuples, langues et cultures autochtones), système éducatif (université, écoles, édition des manuels), domaine médiatique (presse, radio, télévision), nouvelles technologies. Nous avons eu l'impression d'être en présence d'un véritable réseau dont les membres font constamment le lien entre l'enseignement, les médias et la recherche d'une part, et les usagers de la langue, quel que soit leur niveau de compétence. Ceux-ci se réunissent même régulièrement dans un club de discussion, véritable lieu de vie pour le vepse oral (cf. figure 1 infra) :

Figure 1

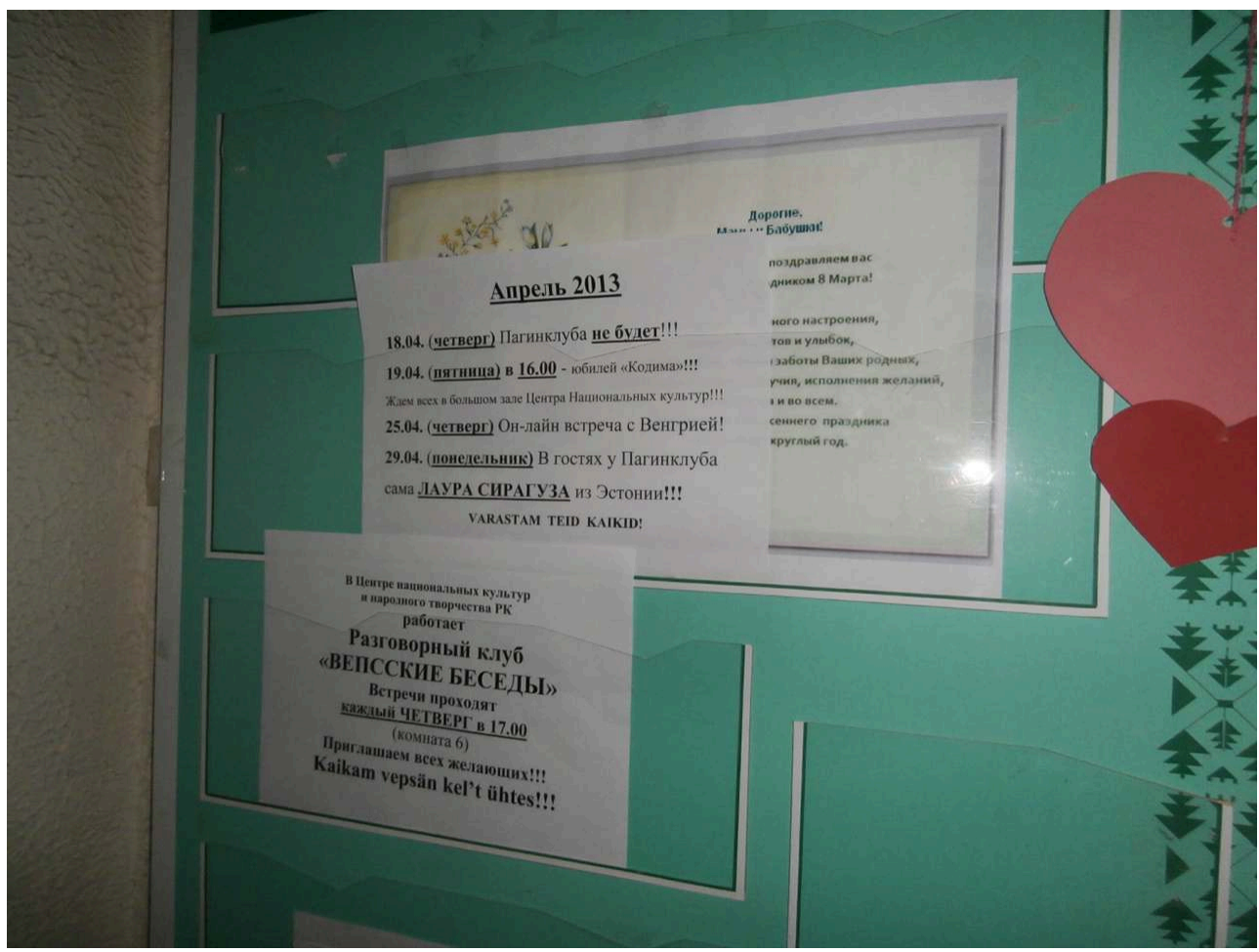

Affiche devant le Paginklub (club de discussion). Petrozavodsk, avril 2013.

Photo K. Djordjevic Léonard.

Concernant les locuteurs, nous avons rencontré sur place un peu de tout: quelques locuteurs traditionnels, dans les villages situés sur la rive du lac Onega, pour certains de véritables virtuoses de la langue; beaucoup de semi-locuteurs, pour qui notre visite a été parfois l'occasion de pratiquer la langue qu'en réalité ils ont peu l'occasion de parler et dans laquelle ils se sentent parfois en insécurité; des locuteurs fantômes qui nous disaient que parler en vepse ne servait plus à grand-chose et qui ne comprenaient donc pas notre intérêt pour cette langue ; et un certain nombre de néo-locuteurs, dont certaines personnes qui ont précisément saisi au vol l'opportunité du réveil identitaire des peuples finno-ougriens de Russie pour se réapproprier la langue d'origine. Une langue parlée par à peine quelques milliers de personnes est une langue en difficulté avérée, quel que soit le degré de cette vulnérabilité. Mais si parmi ces personnes, il y a une telle variation de locuteurs, au sein desquels dominent les locuteurs traditionnels, pas entièrement acculturés, et des néo-locuteurs très motivés, organisés en réseau, conscients de la chance historique qu'ils ont de faire quelque chose d'utile pour leur langue et leur culture, et ouverts à toute initiative venant de la part 
des chercheurs locaux (et internationaux), la survie de cette langue semble alors moins compromise. La parole, les idées, les matériaux circulent du monde de la recherche vers les locuteurs et vice-versa, chacun apportant sa pierre de touche à cette entreprise complexe d'aménagement linguistique « de par en bas ».

En tant que chercheurs, nous avons été réellement intéressés par tout le travail que ce groupe de personnes, locuteurs-activistes, entreprend au quotidien, émerveillés par la passion qui les motive, au service d'un projet de valorisation de la langue, avec un haut degré de participation et d'implication de tous. Une fois de plus, nous avons compris que la réalité se nichait dans une diversité de situations, d'interactions, de relations, selon le degré d'implication du chercheur, son image, son attitude, sa sensibilité, ses capacités d'observation. C'est cette trame qui constitue le tissu d'observations sociolinguistiques, et sur laquelle on peut appliquer ou construire des modèles théoriques.

\section{Terrain tabarquin}

20 L'année suivante, à la même période, nous sommes partis faire du terrain en Italie, au sein de la communauté tabarquine de Sardaigne. Travaillant sur les langues des Balkans, y compris dans la dimension transfrontalière, nous avons déjà eu l'occasion de nous intéresser aux configurations sociolinguistiques d'Italie, notamment à celle impliquant les minorités balkaniques (Slovènes, Albanais, etc.). En 2014, la perspective de travailler sur une situation sociolinguistique unique, que nous avons connue à travers nos lectures (ex. Toso, 2012), et une population vivant sur une île ensoleillée de la Méditerranée, était un facteur de motivation supplémentaire pour découvrir de nouveaux horizons de recherche.

21 Notre premier travail dans ce domaine était centré sur l'histoire des Tabarquins de Sardaigne en tant que trajectoire d'une minorité qui s'est déplacée à plusieurs reprises à travers le bassin méditerranéen. Il nous a semblé intéressant d'observer à quel point cette migration en ricochet - Pegli, Tabarka, Alicante, îles des Sulcis - est aujourd'hui véritablement intégrée à la mémoire collective des Tabarquins et occupe une place importante dans l'histoire orale et écrite, ou érudite, de cette minorité. Nous avons analysé sa mise en récit, à travers notamment un corpus d'entretiens réalisés avec les locuteurs et les travailleurs culturels et associatifs tabarquins, lors du séjour linguistique de terrain à Carloforte, en avril 2014, et d'un corpus d'articles publiés dans la revue locale Quaderni Tabarchini (2011-2014). Une fois de plus donc, notre choix s'est porté sur l'élite locale, les activistes et les aménageurs de la langue "de par en bas ", dont par exemple les auteurs de manuels scolaires (cf. figure 2 infra) : 


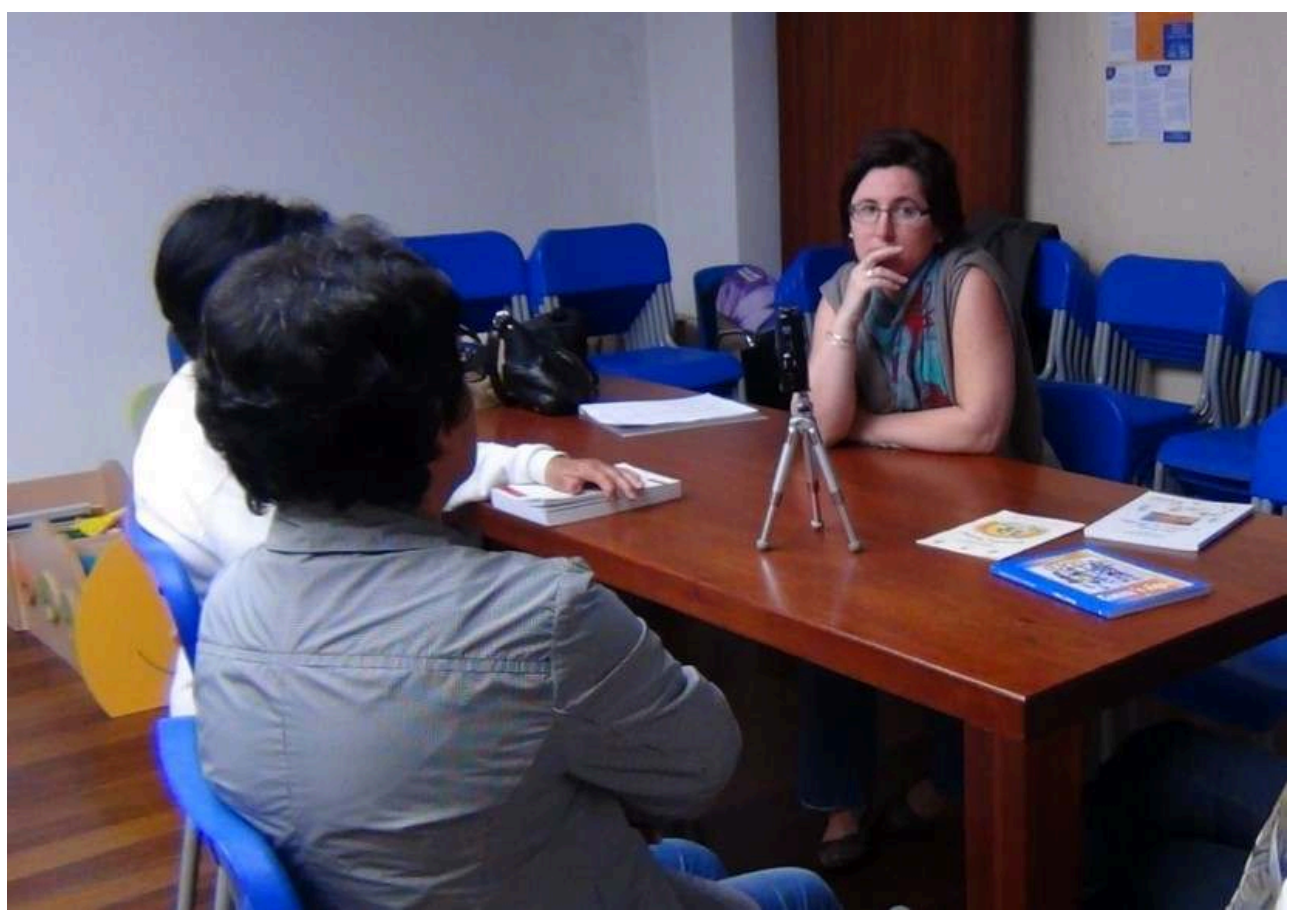

Entretiens avec des activistes tabarquins de Carloforte. Mai 2014.

Photo J. L. Léonard.

Lors des entretiens avec les aménageurs du tabarquin de l'île de San Pietro, nous avons utilisé la méthodologie développée par J. L. Léonard et L. Jagueneau (2013) dans le cadre $\mathrm{du}$ projet «Les langues et vous» (PEPS 2010-2011), qui permet d'interroger les motivations et les modes d'action des activistes dans la promotion de la langue, les conditions dans lesquelles celle-ci se réalise, les objectifs poursuivis et les événements marquants de cette activité. Et tout comme dans le travail sur le vepse, on peut y observer la synergie entre le travail du linguiste et celui de la société civile locale. Ce qui nous a le plus marqué dans les entretiens réalisés c'est une forme de reconnaissance vis-à-vis du linguiste qui a le plus travaillé sur le tabarquin, en l'occurrence Fiorenzo Toso, qui a fixé des règles orthographiques de la langue, écrit sa grammaire, entrepris de faire son dictionnaire. Cette même reconnaissance transparaît dans les écrits du linguiste : s'il a été une caution scientifique pour les locuteurs, les locuteurs ont été sa principale ressource. Il faut dire que le terrain s'y prêtait: le tabarquin est une langue qui n'a pas dit son dernier mot. Des locuteurs traditionnels sont très présents, nous dirions même qu'ils dominent aujourd'hui sur l'île de San Pietro. On n'a pas constaté des attitudes négatives au sein de la population, comme celles que l'on peut trouver chez des locuteurs fantômes de la typologie mentionnée plus haut, ni même l'existence des néo-locuteurs. En réalité, on n'en a pas besoin à San Pietro, tant la langue semble encore vivante et protégée par la volonté de ses habitants.

Nos deux principaux corpus - entretiens transcrits et presse écrite - ont été complétés plus tard par un troisième, secondaire, mais très complémentaire des précédents : plusieurs pages Internet gérées par les membres de la communauté et consacrées à son histoire. Nous avons essayé de montrer en quoi l'approche résiliente de l'histoire et du récit de la migration que l'on observe dans ce corpus contribue à dessiner un véritable 
tableau historique de la mémoire collective. Nous avons retravaillé ce corpus en nous intéressant également à la micro-territorialité et à l'exiguïté dans l'émergence de l'identité insulaire et linguistique des Tabarquins. Il semble, en effet, que c'est précisément l'insularité qui a permis la sauvegarde de l'idiome tabarquin à travers les siècles et malgré les migrations fréquentes d'un territoire ou d'une île à l'autre.

Ainsi procède donc le sociolinguiste sur le terrain : il écoute, enregistre, filme, collecte les données sans savoir forcément ce qui va réellement lui servir une fois de retour chez lui. Un corpus comme celui collecté chez les Tabarquins s'est avéré pour nous une ressource inépuisable, tant il est riche en données, en indices, en enseignements sur le monde du possible, quand une langue minoritaire avec très peu de locuteurs, qui logiquement devrait être très en difficulté, est protégée par ses propres locuteurs, et résiste de manière spectaculaire à l'assimilation, à la fois en raison de l'insularité, mais aussi par la densité démographique de ses locuteurs, fortement majoritaires dans l'île, et peu enclins à « s'expatrier ».

\section{Terrain croate}

Le terrain réalisé sans encombre dans un lieu enchanteur - à Carloforte - et sur une situation aussi riche en enseignements pour les sociolinguistes, a été pour nous un facteur de motivation supplémentaire pour un autre terrain en Italie, réalisé deux ans plus tard, en 2016, sur la situation sociolinguistique des Croates du Molise. Ce terrain présente des caractéristiques communes avec les deux précédents. Il s'agit également d'une micro communauté qui ne compte plus que quelques centaines de locuteurs, installés dans trois villages de l'Italie méridionale. Cependant, si l'on la compare avec le tabarquin, la langue parlée par les Croates du Molise - le na našu - est d'ores et déjà bien engagée dans le processus d'assimilation par l'italien. Cependant, cette acculturation linguistique semble ici aussi-certes très légèrement-freinée d'une part par la mobilisation de la société civile - encore un exemple des acquis de l'aménagement linguistique «de par en bas »- et d'autre part, par l'intérêt de l'État croate pour sa minorité présente depuis plusieurs siècles sur le sol italien. Le séjour de terrain nous a permis d'observer une sorte de dynamique d'altérités croisées entre le croate molisain, le croate standard, l'italien régional et l'italien standard, mais aussi de découvrir une variété archaïque d'une variété proche de notre langue maternelle, en visitant une enclave dans une région reculée d'un pays étranger proche, ce qui est en soi une expérience originale et stimulante, pour une sociolinguiste.

Une fois sur place, nous avons pu constater la forte implication de la société civile locale à travers des actions visant à permettre l'usage élargi de la langue croate, et sa visibilité dans le domaine public. Ces actions couvrent le domaine médiatique, éditorial, culturel, éducatif. Si l'enseignement dans le primaire est assuré par les initiatives communes venant «de par en haut»-ce qui lui confère d'ailleurs un côté " déconnecté de la réalité » dans la mesure où c'est le croate standard qui est enseigné, et non pas la variété locale -, d'autres initiatives d'enseignement facultatif ont vu le jour. Ces initiatives confèrent à la langue une valeur davantage symbolique que fonctionnelle, et ne freinent que très légèrement le rouleau compresseur de l'assimilation. 
Figure 3

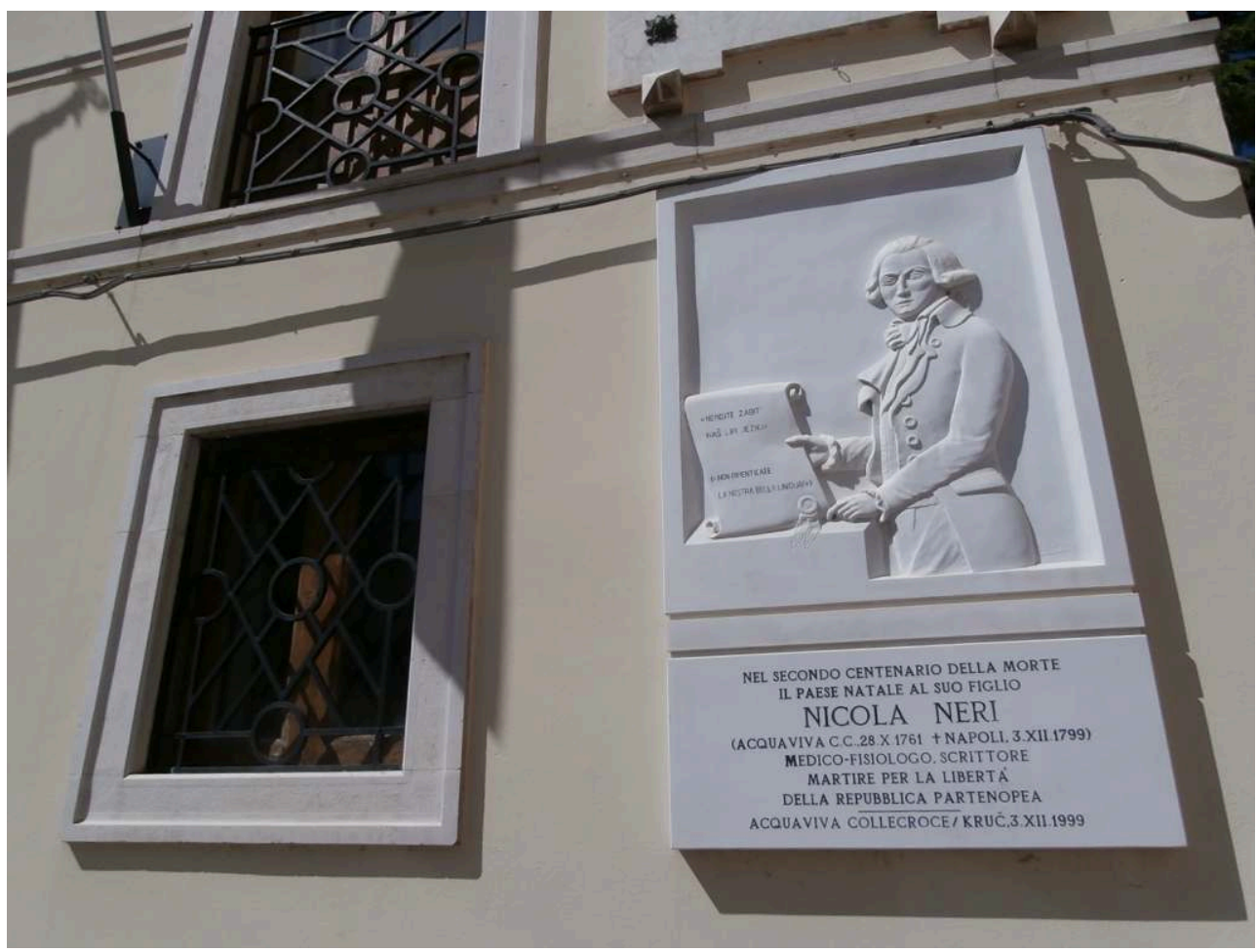

Mairie d'Acquaviva Collecroce. Avril 2016.

Photo K. Djordjevic Léonard.

Lorsque nous sommes arrivés dans la région, nous avions encore en tête le récit pittoresque de Milan Rešetar du début $\mathrm{du} \mathrm{xx}^{\mathrm{e}}$ siècle, décrivant l'inaccessibilité quasi-totale de ces dernières localités croatophones, pratiquement jusqu'à cette époque, tant les infrastructures étaient inexistantes ou en mauvais état (Rešetar, 1911). Aujourd'hui, naturellement, l'isolement de ces trois communes est relatif. Les routes ne sont pas, certes, toujours en très bon état, mais on se rend facilement d'un village à l'autre. Des trois communes, celle qui semble le mieux résister aujourd'hui est celle d'Acquaviva Collecroce (cf. figure 3 supra). Nous y avons fait des rencontres, et réalisé plusieurs entretiens, aussi improbables qu'inattendus. Ainsi, par exemple, nous avons interviewé un employé de la mairie et écrivain qui a publié plusieurs romans bilingues (na našu - italien), et qui s'est avéré un excellent connaisseur aussi bien de l'histoire de sa communauté que de sa ville. Une de ces personnes sincèrement et profondément attachées à leur langue et à leur culture. Une de celles également chez qui on sent un véritable sentiment de tristesse face à l'assimilation galopante qui menace la communauté. Nous avons pu nous entretenir également avec la coordinatrice du "guichet linguistique » d'Acquaviva Collecroce, avec l'enseignante de croate standard, la représentante de la communauté molisaine à Zagreb, ainsi qu'avec un historien local, auteur de nombreux travaux sur la situation du croate molisain. Chez toutes ces personnes, appartenant eux aussi à l'élite locale, sans exception, on sent une volonté de faire perdurer la langue et la culture, malgré l'esprit d'idéologie utilitaire toujours présente, susceptible de compromettre toute action de valorisation des langues minoritaires. 
En séjournant à Acquaviva Collecroce, nous avons pu constater que la grande majorité de la population parlait véritablement la langue, aussi bien dans la rue, que dans les deux cafés que comporte le village. Ces cafés fonctionnent également comme des lieux de socialisation : la préparation de la fête de Maja, à travers les chants traditionnels, accompagnés d'un accordéon, a également lieu dans les cafés et les rues du village. Mais force est de constater que les locuteurs effectifs (locuteurs traditionnels) sont aujourd'hui plutôt âgés, et que les jeunes générations semblent avoir opté pour l'usage de l'italien au quotidien, avec, tout au mieux, des éléments d'alternance codique (ce qui fait d'eux des semi-locuteurs ou même des sous-locuteurs), qui tendent, cela dit, à s'effacer chez les plus jeunes. Dans l'un des trois villages croatophones, nous avons même entendu évoquer les derniers locuteurs du dialecte local...

Une autre remarque pour terminer : pour l'oreille d'une locutrice du serbo-croate, ce terrain fut une grande découverte en soi, notamment à cause de la variété linguistique en usage. Le na našu sonne véritablement comme une variété slave italianisée. On se souvient là aussi des mots de Milan Rešetar : « la première impression que ce dialecte doit faire à chaque locuteur serbocroatophone est d'avoir devant soi les Italiens qui mâchent mal le serbo-croate » (Rešetar, 1911, p. 88). En réalité, le na našu est un dialecte croate à part entière, aucunement "créolisé ", et dont la finesse des contrastes grammaticaux force l'admiration.

\section{Conclusion}

30 La sociolinguistique, depuis sa constitution en tant que discipline, étudie les données observables, en prenant en compte les rapports sociaux existants. Pour les sociolinguistes, «le langage [est] considéré comme une activité, socialement localisée, et dont l'étude se mène sur le terrain » (Bachmann et al., 1981, p. 30). Dans cette contribution, nous avons essayé de montrer que le terrain n'était pas donné d'avance, il se construit entre le linguiste et les locuteurs: «le terrain n'est pas un territoire à disposition - une mine ou un gisement de ressources en observables - mais un système interactif et, qui plus est, un système tenant davantage du hasard que de la nécessité » (Léonard et Petrović, 2015, p. 254). C'est dans ces jeux de la vie et du hasard que se rencontrent les linguistes, les activistes et les locuteurs, et de leur ouverture, disponibilité, tolérance et résilience naît le terrain.

31 Sur les trois terrains sociolinguistiques retenus ici, ce sont des porteurs des initiatives innovantes et créatives, qui, en terres vepses comme dans l'île de San Pietro en Sardaigne, ou à Acquaviva Collecroce, cultivent un pluralisme socioculturel. Certes, les trois terrains ne se firent pas sans certaines difficultés, sur les plans logistiques, de disponibilité des locuteurs et des activistes, ou en ce qui concerne la pratique de la langue - paradoxalement, nous avons pu enregistrer davantage de vepse que de croate molisain, alors que notre compétence linguistique aurait pu favoriser le second sur le premier... Mais ces aléas n'ont pas empêché de réaliser une collecte unique, de rassembler des témoignages de grande qualité, et de nous sentir bien accueillis et bien reçus dans les trois contextes sociaux mentionnés.

32 Sans doute que le trait commun à ces trois expériences heureuses de terrain tient à un facteur fondamental, qui a guidé aussi bien notre démarche que nos conclusions : la perspective de valorisation de la langue et du travail sur la langue réalisé par nos interlocuteurs, mais aussi leur respect et leur amour pour leur langue, leur culture 
et leur « île »-car, de manières différentes, tantôt métaphorique, tantôt effective sur le plan physique, ces trois terrains - vepse, tabarquin et croate molisain -, sont des îles, au sein d'archipels minoritaires.

On retiendra par ailleurs, sur le plan méthodologique, trois angles d'approche, induits par les formes particulières d'activisme de chacun de ces trois terrains: trame de l'intrication entre les aménageurs couvrant pratiquement toute la gamme de la typologie des locuteurs, dans le cas du vepse; mise en récit de la complexe histoire migratoire locale et récit de l'élaboration du corpus en partenariat avec un éminent linguiste, dans le cas du tabarquin ; enfin, le caractère davantage atomisé de la situation du na našu, mais dont les chevilles ouvrières sont des personnalités hautement créatives, engagées sur de multiples fronts. En somme, on retrouve là une belle illustration de la dimension fondamentale du terrain : la dimension humaine.

\section{BIBLIOGRAPHIE}

BACHMANN Christian, LINDENFELD Jacqueline \& SIMONIN Jacky, 1981, Langage et communications sociales, Hatier, Didier, Crédif, Paris, 223 p.

BEAUD Stéphane \& WEBER Florence, 2003, Guide de l'enquête de terrain, La Découverte, Paris, 356 p. BERT Michel \& GRINEVALD Colette, 2010, « Proposition de typologie des locuteurs de LED » in Faits de Langues, $\mathrm{n}^{\circ} 35-36$, p. 117-132.

BLANCHET Philippe, 2000, Linguistique de terrain - Méthode et théorie. Une approche ethnosociolinguistique, Presses Universitaires de Rennes, Rennes, 145 p.

CALVET Louis-Jean \& DUMONT Pierre (dir.), 1999, L'enquête sociolinguistique, L'Harmattan, Paris, $194 \mathrm{p}$.

CostA James, 2013, « Enjeux sociaux des mouvements de revitalisation linguistique » in Langage \& Société, $\mathrm{n}^{\circ} 145$, p. 7-14.

ELOY Jean-Michel (dir.), 2004, Des langues collatérales. Problèmes linguistiques, sociolinguistiques et glottopolitiques de la proximité linguistique., tome. 2, Des langues collatérales, L'Harmattan, Paris, $652 \mathrm{p}$.

FORLOT Gilles \& MARTIN Fanny (dir.), 2014, Regards sociolinguistiques contemporains : terrains, espaces et complexités de la recherche, L'Harmattan, Paris, $340 \mathrm{p}$.

LÉONARD Jean Léo, 2014, «L'enquête dialectologique, entre documentation linguistique et collectage : Noirmoutier 1980 et $2010 »$, in FORLOT Gilles \& MARTIN Fanny (dir.), Regards sociolinguistiques contemporains : terrains, espaces et complexités de la recherche, L'Harmattan, Paris, p. 57-77.

LÉONARD Jean Léo \& JAGUENEAU Lilianne, 2013, « Disparition, apparition et réapparition des langues d'oïl : de l'invisibilisation au nouveau regard » in Bulletin de la Société de Linguistique de Paris, $\mathrm{n}^{\circ}$ 108, vol. 1, p. 283-343, DOI : 10.2143/BSL.108.1.3019220. 
LÉONARD Jean Léo \& PETROVIC Marijana, 2015, « Cadrage, décadrage et recadrage de l'expérience : le terrain comme vertige existentiel », in AVILÉS GONZÁLEZ Karla Janiré \& LÉONARD Jean Léo (dir.), Documentation et revitalisation des « langues en danger ». Épistémologie et praxis, Michel Houdiard Éditeur, Paris, p. 235-289.

REŠETAR Milan, 1911, Die Serbokroatischen Kolonien Süditaliens, Alfred Hölder (coll. Schriften der Balkankommission), Wien, $402 \mathrm{p}$.

TOSo Fiorenzo, 2012, La Sardegna che non parla sardo. Profilo storico e linguistico delle varietà alloglotte : Gallurese, Sassarese, Maddalenino, Algherese, Tabarchino, CUEC, Cagliari, 189 p.

\section{NOTES}

1. Pour réaliser ces trois terrains, nous avons pu bénéficier d'une modeste aide financière de la part de notre équipe de recherche Dipralang de l'université Montpellier 3 (que nous remercions ici). Aucun de ces trois terrains ne s'inscrit cependant dans un projet de grande envergure ou, du moins, avec financements spécifiques. Cette contrainte matérielle de départ leur a conféré cependant une grande liberté : liberté de choix (thèmes, dates, lieux, informateurs), de déplacement, d'angles d'approches et de méthodes de travail. Les trois terrains ont été par ailleurs réalisés conjointement avec Jean Léo Léonard de l'université Paris-Sorbonne.

2. Nous pouvons signaler ici deux ouvrages collectifs récents qui abordent la question du terrain en linguistique et en sociolinguistique précisément à travers le prisme de la réflexivité du chercheur: Forlot et Martin (dir.), 2014, et Léonard et Janiré Avilés González (dir.), 2015.

\section{RÉSUMÉS}

Trois expériences récentes de terrain réalisées dans un contexte minoritaire européen sont présentées ici, qui illustrent les différentes facettes d'un aménagement linguistique conduit essentiellement par la société civile : les terrains vepse, tabarquin et croate molisain. Après avoir défini la (socio)linguistique de terrain, puis l'aménagement linguistique « de par en bas ", nous relaterons notre expérience de terrain chez les Vepses, les Tabarquins et les Croates du Molise, afin d'illustrer la synergie possible entre les linguistes, les activistes et les locuteurs. Malgré les contradictions ou les difficultés d'ajustement entre milieux socioculturels et objectifs, cette synergie est, selon nous, la clé de la réussite d'un terrain sociolinguistique, et illustre ses dimensions fondamentales $\mathrm{l}$ la dimension humaine et du consensus.

Here, we are going to present some recent experiences in the context of European minorities' activism, which illustrate some aspects of grassroots language planning coming from the civil society: the cases of Vepsian, Tabarquin and Molise Croatian. We start by defining the notions of engaged sociolinguistics as well as grassroots planning, we'll introduce the complicated participant methodology chosen by the Vepsians, the Tabarquin and the Molise Croatian in order to promote their linguistic identity, in synergy between linguist, speakers and activists. We are 
convinced that this synergy is the key of success in an empirical sociolinguistic work; it illustrates the basic aspects of language planning, its humanistic and consensual dimensions.

Esitatakse siinkohal kolm hiljutist välitöökogemust, vepslaste, tabarkiinide ja Molise horvaatide juures. Need kõik puudutavad Euroopa vähemuste konteksti jan ad näidlikustavad tsiviilühiskonna juhitud keeleplaneerimise erinevaid aspekte. Eelkõige defineeritakse välitöö sotsiolingvistikat ja siis keeleplaneerimist « rohutasandil ». Siis kirjeldatakse välitöid vepslaste, tabarkiinide ja Molise horvaatide juures, et näidata kuidas võib toimida koostöö keeleteadlaste, aktivistide ja antud keele kõnelevate kogukonna vahel. Vaatamata vastuolude või kohanemise raskusi sotsiokultuuriliste ja objektiivsete keskondade vahel, see sünergia on autori arvates sotsiolingvistiliste välitöôde õnnestumise võtmeks ja toob välja selle olulised komponendid: inimlik ja konsensuslik aspekti.

\section{INDEX}

Mots-clés : terrain sociolinguistique, aménagement linguistique « de par en bas », linguiste, activisme, minorités, Albanais, Croates du Molise, Italiens, Slovènes, Tabarquins, Vepses, XXIe siècle début, croate, finnois, italien, livvi, lude, serbo-croate, tabarquin, vepse, Acquaviva Collecroce, Alicante, Balkans, Bordeaux, Carélie, Carloforte, Italie, Pegli, Russie, San Pietro Molise, Sardaigne, Sulci, Tabarka, Zagreb

motscleset sotsiolingvistilised välitööd, keeleplaneerimine alt ülesse, keeleteadlane, aktivist, vähemused

Keywords : sociolinguistic fieldwork, language planning downside up, linguist, activist, minorities 